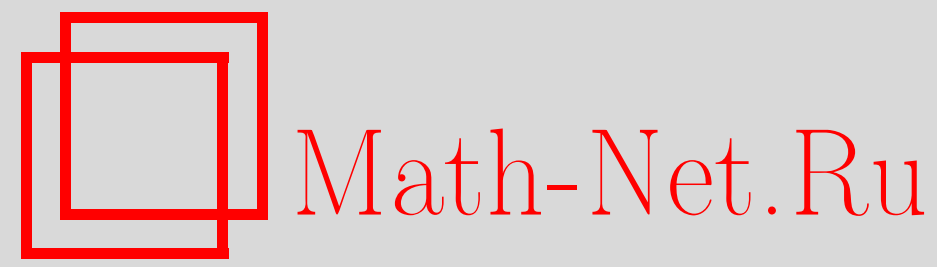

B. Cadre, On a functional version of the convergence of a quadratic form in independent martingales to a $\chi^{2}$ distribution, Теория вероятн. и ее примен., 1998, том 43, выпуск 1, 82-96

DOI: https://doi.org/10.4213/tvp825

Использование Общероссийского математического портала Math-Net.Ru подразумевает, что вы прочитали и согласны с пользовательским соглашением http://www.mathnet.ru/rus/agreement

Параметры загрузки:

IP : 54.162 .127 .20

26 апреля 2023 г., 10:52:47

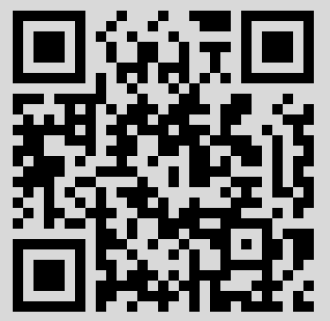




\section{ON A FUNCTIONAL VERSION OF THE CONVERGENCE OF A QUADRATIC FORM IN INDEPENDENT MARTINGALES TO A $\chi^{2}$ DISTRIBUTION}

Пусть $\left(a_{i j}\right)_{i, j \geqslant 1}$ - такая бесконечная вещественная матрица, что $a_{i i}=0$ для любого $i \geqslant 1$, и пусть $\left(X^{i}\right)_{i \geqslant 1}-$ такая последовательность независимых мартингалов, что $\sup _{i \geqslant 1} \mathbf{E}\left[\left(X_{1}^{i}\right)^{4}\right]<\infty$ и для каждого $i \geqslant 1$ предсказуемый компенсатор квадратичной вариации $X^{i}$ есть тождественная функция. В случае, когда $\sigma_{n}^{2}=\sum_{i, j=1}^{n} a_{i j}^{2}$ для каждого $n \geqslant 1$, дано необходимое и достаточное условие того, что процесс, определенный формулой $\sigma_{n}^{-1} \sum_{i<j \leqslant n} a_{i j} X_{t}^{i} X_{t}^{j}$ для каждого $n \geqslant 1$ и $t \geqslant 1$, сходится по распределению к $\left((2 \sqrt{p})^{-1} \sum_{i=1}^{p}\left(\left(B_{t}^{i}\right)^{2}-t\right)\right)_{t \leqslant 1}$, где $p \geqslant 1$ и $B^{1}, \ldots, B^{p}$ есть $p$ независимых стандартных броуновских движений. Кроме того, рассмотрен случай, когда $\left(X^{i}\right)_{i \geqslant 1}$ есть последовательность независимых решений «структурного уравнения».

Ключевые слова и фразы: квадратичные формы, $\chi^{2}$-распределения, функциональные предельные теоремы, мартингалы, стохастическое исчисление, броуновское движение.

\section{Introduction}

We shall assume throughout this paper that $A=\left(a_{i j}\right)_{i, j \geqslant 1}$ is a real symmetric matrix such that $a_{i i}=0$ for each $i \geqslant 1$. For each $n \geqslant 1$, let $A_{n}$ be the $n \times n$ matrix defined by $A_{n}=\left(a_{i j}\right)_{i, j=1, \ldots, n}$ and let $\left(\lambda_{i}^{(n)}\right)_{i=1, \ldots, n}$ be the eigenvalues of $A_{n}$ in decreasing order, i.e., $\lambda_{1}^{(n)} \geqslant \lambda_{2}^{(n)} \geqslant \cdots \geqslant \lambda_{n}^{(n)}$.

Let $X=\left(X^{i}\right)_{i \geqslant 1}$ be a sequence of independent, centered and square integrable random variables. Define, for each $n \geqslant 1$, the quadratic form $Q_{n}(X)$ by

$$
Q_{n}(X)=\frac{1}{\sigma_{n}} \sum_{i<j \leqslant n} a_{i j} X^{i} X^{j}
$$

if $\sigma_{n}^{2}=\sum_{i, j=1}^{n} a_{i j}^{2}$. The study of the limiting behavior in distribution of $Q_{n}(x)$ began with Sevastyanov [11], who determined the class of possible 
limit distributions for quadratic forms in independent Gaussian random variables.

The central limit theorem for the quadratic form $Q_{n}(X)$ has already been dealt with in many papers, with various assumptions on the sequence $X$ (see, for example, [6], [8]).

If the random variables $X^{1}, X^{2}, \ldots$ are identically distributed, it is easy to find some conditions (even necessary and sufficient ones) so that the quadratic form $Q_{n}(X)$ converges to a recentered $\chi^{2}$ distribution with $p$ degrees of freedom. In the direction of convergence to elements of the second Wiener chaos see [9] for a slightly better result, and [2] in the case of random matrices.

In this paper, we shall deal with convergence to $\chi^{2}$ distributions, but our functional approach allows us to consider cases of sequences $X=\left(X^{i}\right)_{i \geqslant 1}$ of random variables not necessarily identically distributed. Moreover, the conditions obtained are necessary and sufficient.

In [1], we study the case of Gaussian $U$-statistics, i.e., the sequence $X$ is a sequence of independent random variables with common distribution $\mathcal{N}(0,1)$, and the sum is defined for each $n \geqslant 1$ by $\sum_{i, j \leqslant n} h_{i j}^{(n)}\left(X^{i}, X^{j}\right)$, where, for each $i, j \leqslant n, h_{i j}^{(n)}: \mathbf{R}^{2} \rightarrow \mathbf{R}$ is a twice continuously differentiable function, but only reaching sufficient conditions.

The first part is dedicated to fixing notation; hypotheses and the presentation of the main results (Theorems 1,2 , and 3 ). Then, we will expose some theoretical background, while in the third part we set out the proofs. Finally, in Part IV, we apply Theorem 1 to quadratic forms in independent solutions of the «Structure Equation».

\section{Notation, hypotheses and the main results}

In the following, $\mathbf{D}$ is the space of right-continuous with left-hand limits functions $f:[0,1] \rightarrow \mathbf{R}$ so that $f(0)=0$, endowed with the $J^{1}$-Skorokhod topology. The Borel $\sigma$-field of $\mathbf{D}$ is denoted $\mathscr{D}$. All processes are assumed to be $\mathbf{D}$-valued, i.e., if $Z$ is any process on the probability space $(\Omega, \mathscr{F}, \mathbf{P})$, $Z \in \mathbf{D}$ (P-a.s.) (in particular, (P-a.s.)). Moreover, $\mathscr{L}(Z)$ is the law of $Z$.

Consider a sequence $\left(Z_{n}\right)_{n}$ of processes. We say that $\left(Z_{n}\right)_{n}$ converges in distribution to $Z$, and write $Z_{n} \stackrel{\text { (d) }}{\longrightarrow} Z$, if $\mathscr{L}\left(Z_{n}\right) \rightarrow \mathscr{L}(Z)$ weakly in the space of all probability measures on $(\mathbf{D}, \mathscr{D})$.

Let $Z$ be a square integrable martingale defined on the probability space $(\Omega, \mathscr{F}, \mathbf{P})$. As usual (c.f. [3] or [5]), we denote by $[Z, Z]$ the quadratic variation of $Z,\langle Z, Z\rangle$ the predictable compensator of $[Z, Z]$ and $\Delta Z_{t}$ the jump of $Z$ at $t \leqslant 1$, i.e., $\Delta Z_{t}=Z_{t}-Z_{t-}$. Moreover, if $\nu$ denotes the predictable compensator of the jump measure of $Z$, we write $f(x) * \nu_{t}$ for $\int_{\mathbf{R}} f(x) \nu(d x,[0, t])$, if $t \leqslant 1$.

Consider a sequence $\left(Y_{n}\right)_{n}$ of random variables. The convergence of the sequence $\left(Y_{n}\right)_{n}$ to $Y$ in distribution (respectively in probability) is denoted 
(as for the case of processes) $Y_{n} \stackrel{\text { (d) }}{\longrightarrow} Y$ (respectively $Y_{n} \stackrel{\mathbf{P}}{\longrightarrow} Y$ ).

Finally, let $d \in \mathbf{N}^{*}$. If $M=\left(m_{i j}\right)_{i, j=1, \ldots, d}$ is a $d \times d$ real-valued matrix, let $\|M\|$ be its Hilbert-Schmidt norm, i.e., $\|M\|^{2}=\sum_{i, j=1}^{d} m_{i j}^{2}$. Moreover, $I_{d}$ is the $d \times d$ identity matrix.

Now, we introduce several assumptions on $A$. Let (Ro) be the condition introduced by Rotar' [10]:

$$
\text { (Ro): } \frac{1}{\sigma_{n}^{2}} \max _{i=1, \ldots, n} \sum_{j=1}^{n} a_{i j}^{2} \longrightarrow 0, \quad n \rightarrow \infty,
$$

for $p \in \mathbf{N}^{*},(\mathrm{No})(p)$ the condition:

$$
(\mathrm{No})(p):\left\|\frac{A_{n}}{\left\|A_{n}\right\|}\left(\frac{A_{n}}{\left\|A_{n}\right\|}-\frac{1}{\sqrt{p}} I_{n}\right)\right\| \longrightarrow 0, \quad n \rightarrow \infty,
$$

and the spectral condition $(\mathrm{Sp})(p)$ :

$$
\begin{aligned}
(\mathrm{Sp})(p): \max _{k=1, \ldots, p}\left|\frac{\lambda_{k}^{(n)}}{\sigma_{n}}-\frac{1}{\sqrt{p}}\right| \longrightarrow 0 \quad \text { and } \\
\frac{1}{\sigma_{n}} \max _{k=p+1, \ldots, n}\left|\lambda_{k}^{(n)}\right| \longrightarrow 0, \quad n \rightarrow \infty .
\end{aligned}
$$

$\mathrm{R} \mathrm{e} \mathrm{m} \mathrm{a} \mathrm{r} \mathrm{k}$. If there exists a sequence $\left(c_{i}\right)_{i \geqslant 1}$ such that for each $i, j \geqslant 1$, $a_{i j}=c_{i} c_{j}$ and $a_{i i}=0$, then (No)(1) is satisfied.

$\mathrm{N}$ o t a t i o n. In the following, $p \in \mathbf{N}^{*}$ and $B^{1}, \ldots, B^{p}$ is a sequence of independent standard Brownian motions on $(\Omega, \mathscr{F}, \mathbf{P})$.

Basically, our idea is to introduce a functional point of view in the quadratic form defined in introduction. We then have our main result:

Theorem 1. Let $\left(X^{i}\right)_{i \geqslant 1}$ be a sequence of independent square integrable martingales on the probability space $(\Omega, \mathscr{F}, \mathbf{P})$ such that $\forall i \geqslant 1$ and $\forall t \geqslant 1,\left\langle X^{i}, X^{i}\right\rangle_{t}=t$. For each $t \leqslant 1$, put $X_{t}=\left(X_{t}^{i}\right)_{i \geqslant 1}$ and assume that $\sup _{i \geqslant 1} \mathbf{E}\left[\left(X_{1}^{i}\right)^{4}\right]<\infty$ and (Ro) are satisfied. Then the following assertions are equivalent:

i) $(\mathrm{No})(p)$ is satisfied;

ii) $\left(Q_{n}\left(X_{t}\right)\right)_{t \leqslant 1}$ converges in distribution to

$$
\left(\frac{1}{\sqrt{p}} \sum_{i=1}^{p} \int_{0}^{t} B_{s}^{i} d B_{s}^{i}\right)_{t \leqslant 1}
$$

iii) There exists a function $f \in L^{2}([0,1])$ such that $\left(Q_{n}\left(X_{t}\right)\right)_{t \leqslant 1}$ converges in distribution to

$$
\left(\frac{1}{\sqrt{p}} \sum_{i=1}^{p} \int_{0}^{t} f(s) d B_{s}^{i} \int_{0}^{s} f(u) d B_{u}^{i}\right)_{t \leqslant 1} .
$$


$\mathrm{R} \mathrm{e} \mathrm{m} \mathrm{a} \mathrm{r} \mathrm{k.} \mathrm{The} \mathrm{equivalence} \mathrm{of} \mathrm{Assertions} \mathrm{ii)} \mathrm{and} \mathrm{iii)} \mathrm{means} \mathrm{that} \mathrm{if}$ $\left(Q_{n}\left(X_{t}\right)\right)_{t \leqslant 1}$ converges in distribution to a process of the form

$$
\left(\frac{1}{\sqrt{p}} \sum_{i=1}^{p} \int_{0}^{t} f(s) d B_{s}^{i} \int_{0}^{s} f(u) d B_{u}^{i}\right)_{t \leqslant 1},
$$

then, necessarily, $|f| \equiv 1$. This remark also holds for Theorem 2 .

The Brownian case has its own interest: even without assuming (Ro), we obtain necessary and sufficient conditions.

Theorem 2. Let $\left(W^{i}\right)_{i \geqslant 1}$ be a sequence of independent standard Brownian motions on the probability space $(\Omega, \mathscr{F}, \mathbf{P})$. Put $W_{t}=\left(W_{t}^{i}\right)_{i \geqslant 1}$ if $t \leqslant 1$. Then the following assertions are equivalent:

i) $(\mathrm{No})(p)$ is satisfied;

ii) $Q_{n}\left(W_{1}\right)$ converges in distribution to $(1 / \sqrt{p}) \sum_{i=1}^{p} \int_{0}^{1} B_{s}^{i} d B_{s}^{i}$;

iii) $\left(Q_{n}\left(W_{t}\right)\right)_{t \leqslant 1}$ converges in distribution to

$$
\left(\frac{1}{\sqrt{p}} \sum_{i=1}^{p} \int_{0}^{t} B_{s}^{i} d B_{s}^{i}\right)_{t \leqslant 1}
$$

iv) There exists a function $f \in L^{2}([0,1])$ such that $\left(Q_{n}\left(W_{t}\right)\right)_{t \leqslant 1}$ converges in distribution to

$$
\left(\frac{1}{\sqrt{p}} \sum_{i=1}^{p} \int_{0}^{t} f(s) d B_{s}^{i} \int_{0}^{s} f(u) d B_{u}^{i}\right)_{t \leqslant 1} .
$$

More generally, we state, without proof, the following result for quadratic forms in independent identically distributed martingales. It is an easy consequence of Theorem 2, Rotar's invariance principle (see [10]) enlarged to finite dimensional repartitions of a martingale and Aldous criterion for tightness. In contrast to Theorem 1 , in Theorem 3 , functional convergence is equivalent to pointwise convergence.

Theorem 3. Let $\left(X^{i}\right)_{i \geqslant 1}$ be a sequence of independent identically distributed and square integrable martingales on the probability space $(\Omega, \mathscr{F}, \mathbf{P})$ such that $\forall i \geqslant 1$ and $\forall t \geqslant 1,\left\langle X^{i}, X^{i}\right\rangle_{t}=t$. Put $X_{t}=\left(X_{t}^{i}\right)_{i \geqslant 1}$ if $t \leqslant 1$ and assume (Ro). Then the following assertions are equivalent:

i) $(\mathrm{No})(p)$ is satisfied;

ii) $Q_{n}\left(X_{1}\right)$ converges in distribution to

$$
\frac{1}{\sqrt{p}} \sum_{i=1}^{p} \int_{0}^{1} B_{s}^{i} d B_{s}^{i}
$$

iii) $\left(Q_{n}\left(X_{t}\right)\right)_{t \leqslant 1}$ converges in distribution to

$$
\left(\frac{1}{\sqrt{p}} \sum_{i=1}^{p} \int_{0}^{t} B_{s}^{i} d B_{s}^{i}\right)_{t \leqslant 1} .
$$


Finally, the aim of the next deterministic result is to characterize Condition (No) $(p)$ in terms of the eigenvalues of $A_{n}$.

Proposition 1. Conditions $(\mathrm{No})(p)$ and $(\mathrm{Sp})(p)$ are equivalent.

\section{Theoretical background on the convergence of martingales}

Let $\mathbf{C}=\mathbf{C}([0,1] ; \mathbf{R})$ be the space of continuous functions $f:[0,1] \rightarrow \mathbf{R}$ with $f(0)=0$, endowed with the uniform convergence topology, and $\mathscr{C}$ its Borel $\sigma$-field. For $a \geqslant 0$, let $S_{a}$ be the function $S_{a}: \mathbf{D} \longrightarrow[0,1] \cup\{+\infty\}$ defined, for each $\alpha \in \mathbf{D}$, by

$$
S_{a}(\alpha)=\left\{\begin{array}{l}
\inf \left\{t \leqslant 1:|\alpha(t)| \geqslant a \text { or }\left|\alpha\left(t^{-}\right)\right| \geqslant a\right\}, \\
+\infty \text { if }|\alpha(t)|<a \text { for each } t \leqslant 1 .
\end{array}\right.
$$

Let $\left(Z_{n}\right)_{n}$ be a sequence of martingales defined on the filtered space $\left(\Omega, \mathscr{F},\left(\mathscr{F}_{t}\right)_{t \leqslant 1}, \mathbf{P}\right)$. For each $n \geqslant 1$, let $\nu^{n}$ be the predictable compensator of the jump measure of $Z_{n}$. Denote by $Z$ the canonical process on the filtered space $(\mathbf{C}, \mathscr{C}, \overline{\mathbf{P}}),\left(\mathscr{C}_{t}\right)_{t \leqslant 1}$ the canonical filtration and let $V: \mathbf{D} \rightarrow \mathbf{D}$ be a functional.

The following result can be obtained from [5, Ch. IX, Theorem 4.47] by localization (see [5, Ch. IX, Theorem 3.39] for a similar generalization).

Theorem 4. Assume that

i) $\overline{\mathbf{P}}$ is a unique probability measure such that for each stopping time $T, Z^{T}$ is a continuous martingale, vanishing at 0 and with quadratic variation $V^{T}$;

ii) For each $a>0$ and $\alpha \in \mathrm{D}$, there is a non-decreasing, continuous and deterministic (i.e., independent of $\alpha$ ) function $F_{a}$ such that $\left(F_{a}(t)-\right.$ $\left.\left(V_{t \wedge S_{a}}\right) \circ \alpha\right)_{t \leqslant 1}$ is non-decreasing;

iii) For each $t \leqslant 1$, the application $\alpha \mapsto V_{t}(\alpha)$ is continuous;

iv) For each $\varepsilon>0$

$$
\lim _{a \nearrow \infty} \limsup _{n} \mathbf{P}\left\{|x| I_{\{|x|>a\}} * \nu_{1}^{n} \geqslant \varepsilon\right\}=0
$$

v) For each $t \leqslant 1, a>0$ :

$$
\left[Z_{n}, Z_{n}\right]_{t \wedge S_{a}\left(Z_{n}\right)}-\left(V_{t \wedge S_{a}}\right) \circ Z_{n} \stackrel{\mathbf{P}}{\longrightarrow} 0, \quad n \rightarrow \infty .
$$

Then, the laws $\mathscr{L}\left(Z_{n}\right)$ converge weakly towards $\overline{\mathbf{P}}$.

The following result, due to [5, Ch. IX, Theorem $4.47 \mathrm{~b}]$, is a converse of Theorem 4:

Theorem 5. Assume that the laws $\mathscr{L}\left(Z_{n}\right)$ converge weakly towards $\overline{\mathbf{P}}$, and that Assertions i), iii) and iv) of Theorem 4 are satisfied. Then, for each $t \leqslant 1$ :

$$
\left[Z_{n}, Z_{n}\right]_{t}-V_{t}\left(Z_{n}\right) \stackrel{\mathbf{P}}{\longrightarrow} 0, . n \rightarrow \infty .
$$




\section{Proofs}

In this section, we adopt the notation of Part II, with

$$
\overline{\mathbf{P}}=\mathbf{P} \circ\left(\left(\frac{1}{\sqrt{p}} \sum_{i=1}^{p} \int_{0}^{t} B_{\mathrm{s}}^{i} d B_{\mathrm{s}}^{i}\right)_{t \leqslant 1}\right)^{-1} .
$$

The process $Z$ will be the canonical process on the filtered space $\left(\mathbf{C}, \mathscr{C},\left(\mathscr{C}_{t}\right)_{t \leqslant 1}, \overline{\mathbf{P}}\right)$. For each $n \geqslant 1$, the martingale $Z_{n}$ will be $\left(Q_{n}\left(X_{t}\right)\right)_{t \leqslant 1}$ for the proof of Theorem 1, and $\left(Q_{n}\left(W_{t}\right)\right)_{t \leqslant 1}$ for the proof of Theorem 2 . For the sake of convenience, we put

$$
Q_{n}(X)=\left(Q_{n}\left(X_{t}\right)\right)_{t \leqslant 1} \quad \text { and } \quad Q_{n}(W)=\left(Q_{n}\left(W_{t}\right)\right)_{t \leqslant 1}
$$

Finally, $V$ will be the functional

$$
V: \mathbf{D} \rightarrow \mathbf{C}, \quad \alpha \mapsto \int_{0}^{\bullet}\left(\frac{2}{\sqrt{p}} \alpha(s)+s\right) d s .
$$

First of all, we state two lemmas, delaying their proofs to the end of this section.

Lemma 1. Assertions i), ii) and iii) of Theorem 4 are satisfied by the functional $V$.

Lemma 2. Let $X$ be a square integrable martingale on the space $\left(\Omega, \mathscr{F},\left(\mathscr{F}_{t}\right)_{t \leqslant 1}, \mathbf{P}\right)$ such that $\mathbf{E}\left[X_{1}^{2}\right]=1$, and $\nu$ the predictable compensator of the jump measure of $X$. Then, for $a>0$ and $\varepsilon>0$ :

$$
\mathbf{P}\left\{|x| I_{\{|x|>a\}} * \nu_{1} \geqslant \varepsilon\right\} \leqslant\left(1+\frac{1}{\varepsilon}\right)\left(\mathbf{P}\left\{\sup _{t \leqslant 1}\left|\Delta X_{t}\right|>a\right\}\right)^{1 / 2} .
$$

The next lemma is derived from [8, Lemma 1.3].

Lemma 3. Let $\left(\eta^{i}\right)_{i \leqslant 1}$ be a sequence of centered independent random variables, and for each $n \geqslant 1, B_{n}=\left(b_{i j}\right)_{i, j=1, \ldots, n}$ a real symmetric matrix with zeros on the diagonal. Then, if $q \geqslant 2$, there exists a constant $c$ such that for each $n \geqslant 1$ :

$$
\mathbf{E}\left[\left|\sum_{i, j=1}^{n} b_{i j} \eta^{i} \eta^{j}\right|^{q}\right] \leqslant c \sup _{i \geqslant 1} \mathbf{E}\left[\left|\eta^{i}\right|^{q}\right]^{2}\left\|B_{n}\right\|^{q}
$$

P r o of of $\mathrm{Th}$ e or e $\mathrm{m} 1$. We first prove that i) $\Rightarrow$ ii). According to Lemma 1, one only needs to show that Assertions iv) and v) of Theorem 4 are satisfied.

We begin with v). For each $a>0$ and $n \geqslant 1$, put

$$
S_{a}^{n}=S_{a}\left(Q_{n}(X)\right) \quad \text { and } \quad M^{n}=\left(\left[X^{n}, X^{n}\right]_{t}-t\right)_{t \leqslant 1} .
$$


Note that $M^{n}$ is a martingale. Using the symmetry of the matrix $A_{n}$, we have for each $n \geqslant 1, t \leqslant 1$ and $a>0$

$$
\begin{aligned}
& \left|\left[Q_{n}(X), Q_{n}(X)\right]_{t \wedge S_{a}^{n}}-V_{t \wedge S_{a}^{n}}\left(Q_{n}(X)\right)\right| \\
& =\mid \sum_{i=1}^{n} \int_{0}^{t \wedge S_{a}^{n}} \frac{1}{\sigma_{n}^{2}}\left(\sum_{j=1}^{n} a_{i j} X_{s-}^{j}\right)^{2} d\left[X^{i}, X^{i}\right]_{s} \\
& \quad-\int_{0}^{t \wedge S_{a}^{n}}\left(\frac{2}{\sigma_{n} \sqrt{p}} \sum_{i<j \leqslant n} a_{i j} X_{s}^{i} X_{s}^{j}+s\right) d s \mid \\
& =\left|\sum_{i=1}^{n} \int_{0}^{t \wedge S_{a}^{n}}\left(\frac{1}{\sigma_{n}^{2}}\left(\sum_{j=1}^{n} a_{i j} X_{s-}^{j}\right)^{2}-\left(\frac{1}{\sigma_{n} \sqrt{p}} \sum_{i, j=1}^{n} a_{i j} X_{s}^{i} X_{s}^{j}+s\right)\right) d s\right| \\
& \quad+\frac{1}{\sigma_{n}^{2}}\left|\sum_{i=1}^{n} \int_{0}^{t \wedge S_{a}^{n}}\left(\sum_{j=1}^{n} a_{i j} X_{s-}^{j}\right)^{2} d M_{s}^{i}\right| \\
& \leqslant \\
& \quad \int_{0}^{1}\left|\frac{1}{\sigma_{n}^{2}} \sum_{i, j=1}^{n} a_{i j}^{2}\left(X_{s-}^{j}\right)^{2}-s\right| d s \\
& \quad+\int_{0}^{1}\left|\frac{1}{\sigma_{n}^{2}} \sum_{i=1}^{n} \sum_{1 \leqslant j \neq j^{\prime} \leqslant n} a_{i j} a_{i j^{\prime}} X_{s-}^{j} X_{s-}^{j^{\prime}}-\frac{1}{\sigma_{n} \sqrt{p}} \sum_{i, j=1}^{n} a_{i j} X_{s}^{i} X_{s}^{j}\right| d s \\
& \quad+\sup _{t \leqslant 1}\left|\frac{1}{\sigma_{n}^{2}} \int_{0}^{t} \sum_{i=1}^{n}\left(\sum_{j=1}^{n} a_{i j} X_{s-}^{j}\right)^{2} d M_{s}^{i}\right| .
\end{aligned}
$$

Taking the expectation of the square of the first term of (1), we obtain:

$$
\begin{aligned}
& \left.\mathbf{E}\left[\left(\int_{0}^{1} \frac{1}{\sigma_{n}^{2}} \sum_{i, j=1}^{n} a_{i j}^{2}\left(X_{s-}^{j}\right)^{2}-s\right) d s\right)^{2}\right] \\
& \leqslant \frac{1}{\sigma_{n}^{4}} \int_{0}^{1} d s \mathrm{E}\left[\left(\sum_{j=1}^{n}\left(\left(X_{s-}^{j}\right)^{2}-s\right) \sum_{i=1}^{n} a_{i j}^{2}\right)^{2}\right] \\
& =\frac{1}{\sigma_{n}^{4}} \int_{0}^{1} \sum_{j=1}^{n} \mathrm{E}\left[\left(\left(X_{s-}^{j}\right)^{2}-s\right)^{2}\right] d s\left(\sum_{i=1}^{n} a_{i j}^{2}\right)^{2} \\
& \leqslant\left(\sup _{j \geqslant 1} \mathrm{E}\left[\left(X_{1}^{j}\right)^{4}\right]+1\right) \frac{1}{\sigma_{n}^{2}} \max _{j \leqslant n} \sum_{i=1}^{n} a_{i j}^{2},
\end{aligned}
$$

the rightmost term vanishing, as $n \rightarrow \infty$, according to (Ro). Taking the 
expectation of the square of the second term of (1), we get

$$
\begin{gathered}
\mathbf{E}\left[\left(\int_{0}^{1}\left|\frac{1}{\sigma_{n}^{2}} \sum_{i=1}^{n} \sum_{1 \leqslant j \neq j^{\prime} \leqslant n} a_{i j} a_{i j^{\prime}} X_{s-}^{j} X_{s-}^{j^{\prime}}-\frac{1}{\sigma_{n} \sqrt{p}} \sum_{i, j=1}^{n} a_{i j} X_{s}^{j} X_{s}^{j}\right| d s\right)^{2}\right] \\
\leqslant \int_{0}^{1} \mathbf{E}\left[\left(\frac{1}{\sigma_{n}^{2}} \sum_{i=1}^{n} \sum_{1 \leqslant j \neq j^{\prime} \leqslant n} a_{i j} a_{i j^{\prime}} X_{s}^{j} X_{s}^{j^{\prime}}-\frac{1}{\sigma_{n} \sqrt{p}} \sum_{i, j=1}^{n} a_{i j} X_{s}^{j} X_{s}^{j}\right)^{2}\right] d s,
\end{gathered}
$$

since $\Delta X_{s}^{i}=0$ (P-a.s.) for each $i \geqslant 1$ and $s \leqslant 1$. Then, the last term equals

$$
\begin{gathered}
\int_{0}^{1} \mathbf{E}\left[\left(\frac{1}{\sigma_{n}^{2}} \sum_{1 \leqslant j \neq j^{\prime} \leqslant n} X_{s}^{j} X_{s}^{j^{\prime}}\left(\sum_{i=1}^{n} a_{j i} a_{i j^{\prime}}-\frac{\sigma_{n}}{\sqrt{p}} a_{j j^{\prime}}\right)\right)^{2}\right] d s \\
=\int_{0}^{1} s^{2} d s \frac{2}{\sigma_{n}^{4}} \sum_{1 \leqslant j \neq j^{\prime} \leqslant n}\left(\sum_{i=1}^{n} a_{j i} a_{i j^{\prime}}-\frac{\sigma_{n}}{\sqrt{p}} a_{j j^{\prime}}\right)^{2} .
\end{gathered}
$$

Assuming (Ro), this converges to 0 if and only if

$$
\frac{1}{\sigma_{n}^{4}} \sum_{j, j^{\prime}=1}^{n}\left(\sum_{i=1}^{n} a_{j i} a_{i j^{\prime}}-\frac{\sigma_{n}}{\sqrt{p}} a_{j j^{\prime}}\right)^{2}=\left\|\frac{A_{n}}{\left\|A_{n}\right\|}\left(\frac{A_{n}}{\left\|A_{n}\right\|} \frac{1}{\sqrt{p}} I_{n}\right)\right\|^{2}
$$

converges to 0 , which is satisfied under $(\mathrm{No})(p)$. We now turn to the last term of (1). According to the Burkhölder inequality, there exists a constant $c_{1}$ such that for each $n \geqslant 1$

$$
\begin{aligned}
\mathbf{E} & {\left[\sup _{t \leqslant 1}\left(\frac{1}{\sigma_{n}^{2}} \int_{0}^{t} \sum_{i=1}^{n}\left(\sum_{j=1}^{n} a_{i j} X_{s-}^{j}\right)^{2} d M_{s}^{i}\right)^{2}\right] } \\
& \leqslant \frac{c_{1}}{\sigma_{n}^{4}} \sum_{i=1}^{n} \mathbf{E}\left[\sup _{s \leqslant 1}\left(\sum_{j=1}^{n} a_{i j} X_{s}^{j}\right)^{4}\left[M^{i}, M^{i}\right]_{1}\right] \\
& =\frac{c_{1}}{\sigma_{n}^{4}} \sum_{i=1}^{n} \mathbf{E}\left[\sup _{s \leqslant 1}\left(\sum_{j=1}^{n} a_{i j} X_{s}^{j}\right)^{4}\right] \mathbf{E}\left[\left[M^{i}, M^{i}\right]_{1}\right] .
\end{aligned}
$$

This last equality comes from the fact that for each $i \geqslant 1$, the sum $\sum_{j=1}^{n} a_{i j} X^{j}$ does not depend on $X^{i}$, so that the processes $\left[M^{i}, M^{i}\right]$ and $\sum_{j=1}^{n} a_{i j} X^{j}$ are independent. Then, according to the Burkhölder and Minkowski inequalities, there exists a constant $c_{2}$ such that $\forall n \geqslant 1$ :

$$
\mathrm{E}\left[\sup _{s \leqslant 1}\left(\sum_{j=1}^{n} a_{i j} X_{s}^{j}\right)^{4}\right] \leqslant c_{2} \mathrm{E}\left[\left(\sum_{j=1}^{n} a_{i j}^{2}\left(X_{1}^{j}\right)^{2}\right)^{2}\right]
$$




$$
\leqslant c_{2}\left(\sum_{j=1}^{n} a_{i j}^{2} \mathbf{E}\left[\left(X_{1}^{j}\right)^{4}\right]^{1 / 2}\right)^{2} \leqslant c_{2} \sup _{j \geqslant 1} \mathbf{E}\left[\left(X_{1}^{j}\right)^{4}\right]\left(\sum_{j=1}^{n} a_{i j}^{2}\right)^{2} .
$$

Now, for each $i \geqslant 1$, by the very definition of $M^{i}$ :

$$
\mathbf{E}\left[\left[M^{i}, M^{i}\right]_{1}\right]=\mathbf{E}\left[\left(\left[X^{i}, X^{i}\right]_{1}-1\right)^{2}\right] \leqslant c_{3}\left(1+\mathbf{E}\left[\left(X_{1}^{i}\right)^{4}\right]\right),
$$

for some constant $c_{3}$ independent of $i$. Consequently, the last term in (1) is bounded by

$$
\begin{gathered}
c_{1} c_{2} c_{3}\left(1+\sup _{j \geqslant 1} \mathbf{E}\left[\left(X_{1}^{i}\right)^{4}\right]\right) \sup _{j \geqslant 1} \mathbf{E}\left[\left(X_{1}^{i}\right)^{4}\right] \frac{1}{\sigma_{n}^{4}} \sum_{i=1}^{n}\left(\sum_{j=1}^{n} a_{i j}^{2}\right)^{2} \\
\leqslant c_{1} c_{2} c_{3}\left(1+\sup _{j \geqslant 1} \mathbf{E}\left[\left(X_{1}^{i}\right)^{4}\right]\right) \sup _{j \geqslant 1} \mathbf{E}\left[\left(X_{1}^{i}\right)^{4}\right] \frac{1}{\sigma_{n}^{4}} \max _{j \leqslant n} \sum_{i=1}^{n} a_{i j}^{2},
\end{gathered}
$$

which vanishes, as $n \rightarrow \infty$, under (Ro).

We now show that Assertion iv) of Theorem 4 is fulfilled. For each $n \geqslant 1$, call $\nu^{n}$ the predictable compensator of the jump measure of $Q_{n}(X)$. According to Lemma 2, for each $n \geqslant 1, \varepsilon>0$ and $a>0$ :

$$
\mathbf{P}\left\{|x| I_{\{|x|>a\}} * \nu_{1}^{n} \geqslant \varepsilon\right\} \leqslant\left(1+\frac{1}{\varepsilon}\right)\left(\mathbf{P}\left\{\sup _{t \leqslant 1}\left|\Delta Q_{n}\left(X_{t}\right)\right|>a\right\}\right)^{1 / 2} .
$$

But for each $n \geqslant 1, \sup _{t \leqslant 1}\left(\Delta Q_{n}\left(X_{t}\right)\right)^{2} \leqslant\left[Q_{n}(X), Q_{n}(X)\right]_{1}(\mathbf{P}$-a.s.) so that, according to the Markov inequality:

$$
\mathbf{P}\left\{|x| I_{\{|x|>a\}} * \nu_{1}^{n} \geqslant \varepsilon\right\} \leqslant\left(1+\frac{1}{\varepsilon}\right) \frac{\mathbf{E}\left[\left[Q_{n}(X), Q_{n}(X)\right]_{1}\right]^{1 / 2}}{a}=\left(1+\frac{1}{\varepsilon}\right) \frac{1}{a} .
$$

Clearly, this implies iv).

We now prove that ii) $\Rightarrow$ i). The process $Q_{n}(X)$ converges in distribution to a continuous process, so that the sequence $\left(Q_{n}(X)\right)_{n}$ is $\mathbf{C}$-tight and

$$
\mathbf{P}\left\{\sup _{t \leqslant 1}\left|\Delta Q_{n}\left(X_{t}\right)\right| \geqslant a\right\} \longrightarrow 0 \quad(\forall a>0), n \rightarrow \infty
$$

according to [5, Ch. VI, Proposition 3.26]. Then, according to Lemma 2 this implies, that Assertion iv) of Theorem 4 is satisfied. Moreover, by Lemma 1, Assertions i) and iii) are satisfied as well, so that, using Theorem 5:

$$
\left[Q_{n}(X), Q_{n}(X)\right]_{1}-V_{1}\left(Q_{n}(X)\right) \stackrel{\mathbf{P}}{\longrightarrow} 0, \quad n \rightarrow \infty
$$

We have already proved that, assuming $\sup _{j \leqslant 1} \mathbf{E}\left[\left(X_{1}^{j}\right)^{4}\right]<\infty$ and (Ro), this convergence implies as $n \rightarrow \infty$

$$
\int_{0}^{1}\left(\frac{1}{\sigma_{n}^{2}} \sum_{i=1}^{n} \sum_{1 \leqslant j \neq j^{\prime} \leqslant n} a_{i j} a_{i j^{\prime}} X_{s}^{j} X_{s}^{j^{\prime}}-\frac{1}{\sigma_{n} \sqrt{p}} \sum_{i, j=1}^{n} a_{i j} X_{s}^{i} X_{s}^{j}\right) d s \stackrel{\mathbf{P}}{\longrightarrow} 0 .
$$


By Lemma 3 and the Caushy-Schwarz Inequality, there exists a constant $c_{4}$ such that for each $n \geqslant 1$ and $s \leqslant 1$ :

$$
\begin{aligned}
& \mathbf{E}\left[\left(\frac{1}{\sigma_{n}^{2}} \sum_{1 \leqslant j \neq j^{\prime} \leqslant n} X_{s}^{j} X_{s}^{j^{\prime}} \cdot \sum_{i=1}^{n} a_{j i} a_{i j^{\prime}}\right)^{2}\right] \\
& \leqslant \frac{c_{4}}{\sigma_{n}^{8}} \sup _{j \geqslant 1} \mathbf{E}\left[\left(X_{1}^{i}\right)^{4}\right]^{2} \sum_{1 \leqslant j \neq j^{\prime} \leqslant n}\left(\sum_{i=1}^{n} a_{j i} a_{i j^{\prime}}\right)^{2} \leqslant c_{4} \sup _{j \geqslant 1} \mathbf{E}\left[\left(X_{1}^{j}\right)^{4}\right]^{2} .
\end{aligned}
$$

Similarly, for another constant $c_{5}$ independent of $n$,

$$
\mathrm{E}\left[\left(\frac{1}{\sigma_{n}} \sum_{i, j=1}^{n} a_{i j} X_{s}^{i} X_{s}^{j}\right)^{4}\right] \leqslant c_{5} \sup _{j \geqslant 1} \mathrm{E}\left[\left(X_{1}^{j}\right)^{4}\right]^{2} .
$$

Hence the convergence in (2) holds in $L^{2}$. For each $n \geqslant 1$, call

$$
b_{j j^{\prime}}=\sum_{i=1}^{n} a_{j i} a_{i j^{\prime}} \quad \text { if } j, j^{\prime} \geqslant 1 .
$$

We have

$$
\begin{aligned}
& \mathbf{E}\left[\left(\int_{0}^{1}\left(\frac{1}{\sigma_{n}^{2}} \sum_{i=1}^{n} \sum_{1 \leqslant j \neq j^{\prime} \leqslant n} a_{i j} a_{i j^{\prime}} X_{s}^{j} X_{s}^{j^{\prime}}-\frac{1}{\sigma_{n} \sqrt{p}} \sum_{i, j=1}^{n} a_{i j} X_{s}^{j} X_{s}^{j}\right) d s\right)^{2}\right] \\
& =\int_{0}^{1} \int_{0}^{1} \mathbf{E}\left[\left(\frac{1}{\sigma_{n}^{2}} \sum_{1 \leqslant j \neq j^{\prime} \leqslant n} X_{s}^{j} X_{s}^{j^{\prime}}\left(b_{j j^{\prime}}-\frac{\sigma_{n}}{\sqrt{p}} a_{j j^{\prime}}\right)\right)\right. \\
& \left.\times\left(\frac{1}{\sigma_{n}^{2}} \sum_{1 \leqslant j \neq j^{\prime} \leqslant n} X_{u}^{j} X_{u}^{j^{\prime}}\left(b_{j j^{\prime}}-\frac{\sigma_{n}}{\sqrt{p}} a_{j j^{\prime}}\right)\right)\right] d u d s \\
& =\int_{0}^{1} \int_{0}^{1} \mathbf{E}\left[\left(\frac{1}{\sigma_{n}^{2}} \sum_{1 \leqslant j \neq j^{\prime} \leqslant n} X_{s \wedge u}^{j} X_{s \wedge u}^{j^{\prime}}\left(b_{j j^{\prime}}-\frac{\sigma_{n}}{\sqrt{p}} a_{j j^{\prime}}\right)\right)^{2}\right] d u d s \\
& =2 \int_{0}^{1} \int_{0}^{1}(s \wedge u)^{2} d u d s \frac{1}{\sigma_{n}^{4}} \sum_{1 \leqslant j \neq j^{\prime} \leqslant n}\left(b_{j j^{\prime}}-\frac{\sigma_{n}}{\sqrt{p}} a_{j j^{\prime}}\right)^{2} .
\end{aligned}
$$

We showed in the first part of the proof, that, assuming (Ro), this last term vanishes, as $n \rightarrow \infty$, if and only if $(\mathrm{No})(p)$ is satisfied, which implies i).

Now we prove ii $\Longleftrightarrow$ iii). Clearly, ii) $\Rightarrow$ iii) with $f \equiv 1$ and one only needs to prove that iii) $\Rightarrow$ ii). According to Lemma 3 , there exists a constant 
$c_{6}$ such that for each $n \geqslant 1$ and $t \leqslant 1$ :

$$
\mathbf{E}\left[\left(\sum_{i<j \leqslant n} a_{i j} X_{t}^{i} X_{t}^{j}\right)^{4}\right] \leqslant c_{6} \sigma_{n}^{4} .
$$

Then, the sequence $\left(Q_{n}\left(X_{t}\right)^{2}\right)_{n}$ is uniformly integrable for each $t \leqslant 1$, from which we deduce that

$$
\begin{aligned}
\frac{t^{2}}{2} & =\mathbf{E}\left[Q_{n}\left(X_{t}\right)^{2}\right] \longrightarrow \frac{1}{p} \mathbf{E}\left[\left(\sum_{i=1}^{p} \int_{0}^{t} f(s) d B_{s}^{i} \int_{0}^{s} f(u) d B_{u}^{i}\right)^{2}\right] \\
& =\int_{0}^{t} f^{2}(s) d s \int_{0}^{s} f^{2}(u) d u, \quad n \rightarrow \infty,
\end{aligned}
$$

so that $t=\int_{0}^{t} f^{2}(s) d s \quad \forall t \leqslant 1$ and $f^{2} \equiv 1$. Consequently, $\left(Q_{n}\left(X_{t}\right)\right)_{t \leqslant 1}$ converges in distribution to $(1 / \sqrt{p})\left(\sum_{i=1}^{p} \int_{0}^{t} \bar{B}_{s}^{i} d \bar{B}_{s}^{i}\right)_{t \leqslant 1}$, where for each $i=$ $1, \ldots, p, \bar{B}^{i}$ is the standard Brownian motion defined for each $s \leqslant 1$ by $\bar{B}_{s}^{i}=\int_{0}^{s} f(u) d B_{u}^{i}$, proving ii).

We now prove Theorem 2.

$\mathrm{P}$ r o of of $\mathrm{T}$ h e o r e $\mathrm{m}$ 2. The equivalence between iv) and iii) was established in Theorem 1 , and iii) $\Rightarrow$ ii) is clear.

We show that i) $\Rightarrow$ iii). Standard arguments imply that for each $n \geqslant 1$ and $t \leqslant 1$,

$$
Q_{n}\left(W_{t}\right)=\frac{1}{2 \sigma_{n}} \sum_{i=1}^{n} \lambda_{i}^{(n)}\left(\left(Y_{i}^{(n)}(t)\right)^{2}-t\right),
$$

where $Y_{1}^{(n)}, \ldots, Y_{n}^{(n)}$ are independent standard Brownian motions. According to Lemma 1 and Proposition 1, one only needs to prove that $(S p)(p)$ implies Assertion v) of Theorem 4. Using relation (3), we have for each $a>0, t \leqslant 1$ and $n \geqslant 1$, if $S_{a}^{n}=S_{a}\left(Q_{n}(W)\right)$ :

$$
\begin{aligned}
& {\left[Q_{n}(W), Q_{n}(W)\right]_{t \wedge S_{a}^{n}}-V_{t \wedge S_{a}^{n}}\left(Q_{n}(W)\right)} \\
& \quad=\int_{0}^{t \wedge S_{a}^{n}} \frac{1}{\sigma_{n}^{2}} \sum_{i=1}^{n}\left(\left(\lambda_{i}^{(n)}\right)^{2}-\frac{\sigma_{n}}{\sqrt{p}} \lambda_{i}^{(n)}\right)\left(\left(Y_{i}^{(n)}(s)\right)^{2}-s\right) d s .
\end{aligned}
$$

The processes $Y_{1}^{(n)}, \ldots, Y_{n}^{(n)}$ being independent, we deduce that for each $n \geqslant p$ :

$$
\begin{gathered}
\mathbf{E}\left[\left(\int_{0}^{t \wedge S_{a}^{n}} \frac{1}{\sigma_{n}^{2}} \sum_{i=1}^{n}\left(\left(\lambda_{i}^{(n)}\right)^{2}-\frac{\sigma_{n}}{\sqrt{p}} \lambda_{i}^{(n)}\right)\left(\left(Y_{i}^{(n)}(s)\right)^{2}-s\right) d s\right)^{2}\right] \\
\leqslant \frac{1}{\sigma_{n}^{4}} \mathbf{E}\left[\left(\sum_{i=1}^{n}\left(\left(\lambda_{i}^{(n)}\right)^{2}-\frac{\sigma_{n}}{\sqrt{p}} \lambda_{i}^{(n)}\right)\left(\left(Y_{i}^{(n)}(1)\right)^{2}-1\right)\right)^{2}\right]
\end{gathered}
$$




$$
\begin{aligned}
= & \frac{2}{\sigma_{n}^{4}} \sum_{i=1}^{n}\left(\lambda_{i}^{(n)}\right)^{2}-\left(\lambda_{i}^{(n)}-\frac{\sigma_{n}}{\sqrt{p}}\right)^{2} \\
\leqslant & 2 \sum_{i=1}^{p}\left(\frac{\lambda_{i}^{(n)}}{\sigma_{n}}\right)^{2}\left(\frac{\lambda_{i}^{(n)}}{\sigma_{n}}-\frac{1}{\sqrt{p}}\right)^{2}+2\left(\frac{1}{\sqrt{p}}+\frac{1}{\sigma_{n}} \max _{i=p+1, \ldots, n}\left|\lambda_{i}^{(n)}\right|\right)^{2} \\
& \times\left(1-\sum_{i=1}^{p}\left(\frac{\lambda_{i}^{(n)}}{\sigma_{n}}\right)^{2}\right)
\end{aligned}
$$

because $\sum_{i=1}^{n}\left(\lambda_{i}^{(n)}\right)^{2}=\sigma_{n}^{2}$. It is clear that under $(\mathrm{Sp})(p)$, the last term vanishes as $n \rightarrow \infty$, implying iii).

Only ii) $\Rightarrow$ i) is left to show. It is a classical exercise to prove, using representation (3), that if $Q_{n}\left(W_{1}\right)$ converges in distribution to

$$
\frac{1}{\sqrt{p}} \sum_{i=1}^{p} \int_{0}^{1} B_{s}^{i} d B_{s}^{i}
$$

then

$$
\max _{i=1, \ldots, n}\left|\frac{\lambda_{i}^{(n)}}{\sigma_{n}}-\lambda_{i}\right| \rightarrow 0, \quad n \rightarrow \infty
$$

where $\lambda_{1}=\cdots=\lambda_{p}=(1 / \sqrt{p})$ and $\lambda_{i}=0$ if $i \geqslant p+1$, the numbers $\left(\lambda_{i}\right)_{i \geqslant 1}$ being arranged in decreasing order. Now, $(\mathrm{No})(p)$ is satisfied according to Proposition 1.

Pro of of Proposition 1 . Note that for each $n \geqslant 1$ :

$$
\left\|\frac{A_{n}}{\left\|A_{n}\right\|}\left(\frac{A_{n}}{\left\|A_{n}\right\|}-\frac{1}{\sqrt{p}} I_{n}\right)\right\|^{2}=\sum_{i=1}^{n}\left(\frac{\lambda_{i}^{(n)}}{\sigma_{n}}\right)^{2}\left(\frac{\lambda_{i}^{(n)}}{\sigma_{n}}-\frac{1}{\sqrt{p}}\right)^{2} .
$$

The last term already appeared in (4): we proved that, under $(\mathrm{Sp})(p)$, this term vanishes, as $n \rightarrow \infty$, hence $(\mathrm{Sp})(p) \Longrightarrow(\mathrm{No})(p)$.

Now, assume $(\mathrm{No})(p)$. Then, according to (5) as $n \rightarrow \infty$

$$
\max _{i=1, \ldots, n}\left(\frac{\lambda_{i}^{(n)}}{\sigma_{n}}\right)^{2}\left(\frac{1}{\sqrt{p}}-\frac{\lambda_{i}^{(n)}}{\sigma_{n}}\right)^{2} \leqslant \sum_{i=1}^{n}\left(\frac{\lambda_{i}^{(n)}}{\sigma_{n}}\right)^{2}\left(\frac{1}{\sqrt{p}}-\frac{\lambda_{i}^{(n)}}{\sigma_{n}}\right)^{2} \longrightarrow 0
$$

so that for each $i \geqslant 1$, the sequence $\lambda_{i}^{(n)} / \sigma_{n}$ converges uniformly to 0 or to $1 / \sqrt{p}$. But for each $n \geqslant 1$, the eigenvalues $\left(\lambda_{i}^{(n)}\right)_{i=1, \ldots, n}$ are arrangèd in the decreasing order and $\sum_{i=1}^{n}\left(\lambda_{i}^{(n)}\right)^{2}=\sigma_{n}^{2}$, implying only two possibilities: either $\lambda_{i}^{(n)} / \sigma_{n}$ converges to $1 / \sqrt{p}$ for each $i=1, \ldots, p$ and $\max _{i=p+1, \ldots, n} \lambda_{i}^{(n)} / \sigma_{n}$ converges to 0 as $n \rightarrow \infty$, or $\max _{i=1, \ldots, n} \lambda_{i}^{(n)} / \sigma_{n}$ van- 
ishes as $n \rightarrow \infty$. Suppose the second case occurs. Then, as $n \rightarrow \infty$

$$
\begin{aligned}
& \sum_{i=1}^{n}\left(\frac{\lambda_{i}^{(n)}}{\sigma_{n}}\right)^{2}\left(\frac{1}{\sqrt{p}}-\frac{\lambda_{i}^{(n)}}{\sigma_{n}}\right)^{2} \\
& \quad=\frac{1}{p} \sum_{i=1}^{n}\left(\frac{\lambda_{i}^{(n)}}{\sigma_{n}}\right)^{2}+\sum_{i=1}^{n}\left(\frac{\lambda_{i}^{(n)}}{\sigma_{n}}\right)^{4}-\frac{2}{\sqrt{p}} \sum_{i=1}^{n}\left(\frac{\lambda_{i}^{(n)}}{\sigma_{n}}\right)^{3}
\end{aligned}
$$

converges to $1 / p$, contradicting $(\mathrm{No})(p)$ : therefore the eigenvalues satisfy the first possibility, i.e., $(\mathrm{Sp})(p)$ is valid.

We now prove Lemma 1 . In [1, Lemma 5], we proved the following result:

Lemma 4. Let $T$ be a stopping time and $p \in \mathbf{N}^{*}$. The following assertions are equivalent:

i) $X^{T}$ is a square integrable continuous martingale such that $X_{0}^{T}=0$ and for each $t \leqslant 1$,

$$
\left\langle X^{T}, X^{T}\right\rangle_{t}=\int_{0}^{t \wedge T}\left(\frac{2}{\sqrt{p}} X_{s}+s\right) d s ;
$$

ii) There exist $p$ independent standard Brownian motions $B^{1}, \ldots, B^{p}$ such that for each $t \leqslant 1$,

$$
X_{t \wedge T}=\frac{1}{\sqrt{p}} \sum_{i=1}^{p} \int_{0}^{t \wedge T} B_{s}^{i} d B_{s}^{i} .
$$

Lemma 1 is a straightforward consequence of Lemma 4.

$\mathrm{P}$ r o of of $\mathrm{L} \mathrm{e} \mathrm{m} \mathrm{m} \mathrm{a} \mathrm{1.} \mathrm{Lemma} 4$ implies Assertion i) of Theorem 4. If $a>0$, let $F_{a}$ be the function defined for each $t \leqslant 1$ by

$$
F_{a}(t)=\int_{0}^{t}\left(2 p^{-1 / 2} a+s\right) d s .
$$

Then $\left(F_{a}(t)-V_{t \wedge S_{a}^{n}} \circ \alpha\right)_{t \leqslant 1}$ is non-decreasing for each $\alpha \in \mathbf{D}$ which shows that Assertion ii) of Theorem 4 is satisfied for the function $F_{a}$. Finally, it is clear that for each $t \leqslant 1$, the function $\alpha \longmapsto V_{t}(\alpha)$ is continuous, implying iii).

$\mathrm{P} r$ o of of $\mathrm{L} \mathrm{e} \mathrm{m} \mathrm{m} \mathrm{a} \mathrm{2.} \mathrm{According} \mathrm{to} \mathrm{the} \mathrm{Lenglart-Rebolledo} \mathrm{in-}$ equality (c.f. [5, Ch. I, Lemma 3.30]), for each $a>0, \varepsilon>0$ and $\eta>0$ :

$$
\begin{aligned}
& \left.\mathbf{P}\left\{|x| I_{\{|x|>a\}} * \nu_{1} \geqslant \varepsilon\right\} \leqslant \frac{\eta}{\varepsilon}+\frac{1}{\varepsilon} \mathrm{E}\left[\sup _{s \leqslant 1}\left|\Delta X_{s}\right| I_{\left\{\sup _{s} \leqslant 1\right.}\left|\Delta X_{s}\right|>a\right\}\right] \\
& +\mathbf{P}\left\{\sum_{s \leqslant 1}\left|\Delta X_{s}\right| I_{\left\{\Delta X_{s} \mid>a\right\}}>\eta\right\} \\
& \leqslant \frac{\eta}{\varepsilon}+\frac{1}{\varepsilon} \mathbf{E}\left[\sup _{s \leqslant 1}\left|\Delta X_{s}\right|^{2}\right]^{1 / 2}\left(\mathbf{P}\left\{\sup _{s \leqslant 1}\left|\Delta X_{s}\right|>a\right\}\right)^{1 / 2}
\end{aligned}
$$




$$
\begin{aligned}
& +\mathbf{P}\left\{\sum_{s \leqslant 1}\left|\Delta X_{s}\right| I_{\left\{\Delta X_{s} \mid>a\right\}}>0\right\} \\
\leqslant & \frac{\eta}{\varepsilon}+\frac{1}{\varepsilon} \mathbf{E}\left[\left(X_{1}\right)^{2}\right]^{1 / 2}\left(\mathbf{P}\left\{\sup _{s \leqslant 1}\left|\Delta X_{s}\right|>a\right\}\right)^{1 / 2}+\mathbf{P}\left\{\sup _{s \leqslant 1}\left|\Delta X_{s}\right|>a\right\} \\
\leqslant & \frac{\eta}{\varepsilon}+\left(1+\frac{1}{\varepsilon}\right)\left(\mathbf{P}\left\{\sup _{s \leqslant 1}\left|\Delta X_{s}\right|>a\right\}\right)^{1 / 2} \cdot
\end{aligned}
$$

Now, letting $\eta \rightarrow 0$, we obtain the lemma.

\section{Application of Theorem 1}

Let $f: \mathbf{R} \rightarrow \mathbf{R}$. We shall say that the martingale $X$ defined on $(\Omega, \mathscr{F}, \mathbf{P})$ is a solution to the structure equation $(\mathrm{SE})(f)$ if for each $t \leqslant 1$ :

$$
[X, X]_{t}=t+\int_{0}^{t} f\left(X_{s-}\right) d X_{s} \quad \text { (P-a.s.). }
$$

Note that $\forall t \leqslant 1,\langle X, X\rangle_{t}=t$. Equation $(\mathrm{SE})(f)$ has been studied by many authors. Meyer [7] showed that $(\mathrm{SE})(f)$ admits a solution if $f$ is continuous, but in general, this solution is not unique (c.f. [4]).

If $p \in \mathbf{N}^{*}$ and $B^{1}, \ldots, B^{p}$ is a sequence of independent standard Brownian motions of $(\Omega, \mathscr{F}, \mathbf{P})$, we show the following corollary.

Corollary 1. For each $i \geqslant 1$, let $f_{i}: \mathbf{R} \rightarrow \mathbf{R}$ be a function such that there exists $K_{i} \geqslant 0$ such that $\forall x \in \mathbf{R}:\left|f_{i}(x)\right| \leqslant K_{i} x^{2}$. We suppose that $\left(X^{i}\right)_{i \geqslant 1}$ is a sequence of independent martingales on $(\Omega, \mathscr{F}, \mathbf{P})$ such that for each $i \geqslant 1, X^{i}$ is a solution to (SE) $\left(f_{i}\right)$. Call $X_{t}=\left(X_{t}^{i}\right)_{i \geqslant 1}$ for each $t \leqslant 1$ and assume that $\sup _{i \geqslant 1} K_{i}<\infty$ and (Ro) are satisfied. Then the following assertions are equivalent:

i) $(\mathrm{No})(p)$ is satisfied;

ii) $\left(Q_{n}\left(X_{t}\right)\right)_{t \leqslant 1}$ converges in distribution to

$$
\left(\frac{1}{\sqrt{p}} \sum_{i=1}^{p} \int_{0}^{t} B_{s}^{i} d B_{s}^{i}\right)_{t \leqslant 1}
$$

$\mathrm{R}$ e $\mathrm{m}$ a $\mathrm{r} \mathrm{k}$. We can apply this result to quadratic forms in mixing of Brownian motions $\left(f_{i} \equiv 0\right)$, compensated Poisson processes $\left(f_{i} \equiv \alpha \neq 0\right)$ of Azéma martingales (case $f_{i}(x)=\alpha x+\beta, \forall x \in \mathbf{R}$, if $\alpha \neq 0, \beta \in \mathbf{R}$ ).

P r o of. According to Theorem 1, one only needs to prove that $\sup _{i \geqslant 1} K_{i}<\infty$ implies $\sup _{i \geqslant 1} \mathbf{E}\left[\left(X_{1}^{i}\right)^{4}\right]<\infty$. By the very definition of 
$X^{i}, i \geqslant 1$, we have for each $t \leqslant 1$ :

$$
\begin{aligned}
\mathbf{E}\left[\left(\left[X^{i}, X^{i}\right]_{t}\right)^{2}\right] \leqslant & 2+2 \int_{0}^{t} \mathbf{E}\left[f_{i}\left(X_{s}^{i}\right)^{2}\right] d s=2+2 \int_{0}^{t} \mathbf{E}\left[f_{i}\left(X_{s}^{i}\right)^{2} I_{\left\{\left|X_{s}^{i}\right| \geqslant 1\right\}}\right] d s \\
& +2 \int_{0}^{t} \mathbf{E}\left[f_{i}\left(X_{s}^{i}\right)^{2} I_{\left\{\left|X_{s}^{i}\right|<1\right\}}\right] d s \\
\leqslant & 2\left(1+K_{i}^{2}\right)+2 K_{i}^{2} \int_{0}^{t} \mathbf{E}\left[\left(X_{s}^{i}\right)^{4}\right] d s .
\end{aligned}
$$

Hence, according to the Burkhölder inequality, there is a constant $c$ such that for each $t \leqslant 1$ and $i \geqslant 1$ :

$$
\mathbf{E}\left[\left(X_{t}^{i}\right)^{4}\right] \leqslant 2 c\left(1+K_{i}^{2}\right)+2 c K_{i}^{2} \int_{0}^{t} \mathbf{E}\left[\left(X_{s}^{i}\right)^{4}\right] d s .
$$

Finally, Gronwall lemma implies that for each $i \geqslant 1$,

$$
\mathbb{E}\left[\left(X_{1}^{i}\right)^{4}\right] \leqslant 2 c\left(1+K_{i}^{2}\right) \exp \left\{2 c K_{i}^{2}\right\}
$$

hence the result.

\section{REFERENCES}

1. Cadre $B$. On the convergence of Gaussian $U$-statistics to recentered $\chi^{2}$ distributions with $p$ degrees of freedom. - J. Multivariate Anal. (to appear).

2. Dehling H., Mikosch T. Random quadratic forms and the bootstrap for $U$-statistics. J. Multivar. Anal., 1994, v. 51, p. 392-413.

3. Dellacherie C., Meyer P. A. Probabilités et potentiel: théorie des martingales. Paris: Hermann, 1975.

4. Emery $M$. On the Azéma martingales. - Lect. Notes Math., 1989, v. 1372, p. 66-87.

5. Жакод Ж., Ширяев А. Н. Предельные теоремы для случайных процессов. Т. 1, 2. М.: Физматлит, 1994.

6. Якубовский $A .$, Мемен $Ж$. Функциональные центральные предельные теоремы для одного класса квадратичных форм от независимых случайных величин. Теория вероятн. и ее примен., 1993, т. 38, в. 3, с. 600-612.

7. Meyer $P$. A. Construction de solutions d'équation de structure. - Lect. Notes Math., 1989, v. 1372 , p. $142-146$.

8. Mikosch T. Functional limit theorems for random quadratic forms. - Stochastic Process. Appl., 1991, v. 37, p. 81-98.

9. Mikosch $T$. A weak invariance principle for weighted $U$-statistics with varying kernels. - J. Multivariate Anal., 1993, v. 47, p. 82-173.

10. Ротарь В. И. Некоторые предельные теоремы для полиномов второй степени. Теория вероятн. и ее примен., 1973, т. XVIII, в. 3, с. 527-546.

11. Севастьянов Б. А. Класс предельных законов распределения квадратичных форм от нормальных случайных величин. - Теория вероятн. и ее примен., 1961, т. VI, в. 3, с. $368-372$. 